\title{
Cavity-enhanced ultrafast two-dimensional spectroscopy using higher-order modes
}

\author{
Thomas K. Allison \\ Stony Brook University, Stony Brook, NY 11794-3400
}

\begin{abstract}
We describe methods using frequency combs and cavities for recording two-dimensional ultrafast spectroscopy signals with high sensitivity. By coupling multiple frequency combs to modes of an optical resonator, cavity-enhanced 2D spectroscopy signals are naturally generated.
\end{abstract}

\section{Introduction}

While physicists have demonstrated exquisite control over gas-phase molecular samples [1], the optical spectroscopy that is performed on these systems is usually much less sophisticated than their solution phase counterparts, due to limitations imposed by the very small optical densities of dilute gases. For matter in condensed phases, coherent, all-optical, third-order spectroscopies using ultrashort pulses, such as transient absorption spectroscopy and 2D spectroscopy, have now emerged as powerful techniques for studying both structure and dynamics [2] and are widely applied to variety of problems in chemistry, physics, biology, and materials science. In contrast, most spectroscopy in molecular beams must employ socalled "action" methods, where absorption of a photon causes a detectable change in the system, such as dissociation, ionization, or fluorescence.

Although difficult, ultrasensitive detection of optical signals from molecular beams (a.k.a. "direct absorption") is possible, and has been used for high resolution static spectroscopy for decades [3,4]. In a recent article [5] we described the extension of ultrasensitive direct absorption techniques to femtosecond time-resolved experiments, reporting cavity-enhanced optical measurements in a dilute molecular beam that are simultaneously ultrasensitive and ultrafast. In this contribution, we will describe how this technology can be applied to perform ultrasensitive 2D spectroscopy. However, instead of simply adapting standard techniques for recording 2D spectra to cavity-enhancement, we describe here a method uniquely enabled by the propagation properties of light in optical cavities.

Using higher-order cavity modes, one can naturally record cavity-enhanced 2D signals by mixing three resonantly-enhanced frequency combs with carrier-envelope offset frequencies $\left(f_{\mathrm{CEO}, 1}, f_{\mathrm{CEO}, 2}, f_{\mathrm{CEO}, 3}\right)$ to generate a fourth resonantly enhanced frequency comb with carrierenvelope offset frequency $f^{(3)}= \pm\left(f_{\mathrm{CEO}, 1}-f_{\mathrm{CEO}, 2}\right)+f_{\mathrm{CEO}, 3}$. The $2 \mathrm{D}$ signal is isolated from background signals via a combination of phase cycling and spatial mode-matching/phase 
matching. Since the three frequency combs can share the same repetition rate and differ only in their carrier-envelope offset frequencies, they can be generated using just one mode-locked laser and fixed-frequency acousto-optic modulators (AOMs). Alternatively, using multiple combs with independent repetition rates can allow for dual comb readout of the signal if high resolution is desired [6]. As in cavity-enhanced transient absorption spectroscopy (CE-TAS) [5], the techniques are generally applicable to the UV, visible, and infrared regimes. Also as in CE-TAS, the signal enhancement scales with the cavity finesse squared, such that even using cavities of modest finesse, large enhancements are obtained. Here, we present a brief summary. Complete details can be found in [7].

\section{Phase cycling of higher-order modes}

Phase cycling using higher-order modes is motivated by the mode structure of optical resonators. In an optical cavity, light pulses in different spatial modes acquire a round trip differential phase shift due to the dependence of the round trip Gouy phase on the HermiteGaussian mode order. In general, if light field $\mathrm{E}_{1}$ is in the $T E M_{t m i}$ mode and light field $\mathrm{E}_{2}$ is in the $\mathrm{TEM}_{\mathrm{rnz}}$ mode, each round trip they acquire a phase shift $\left.\Delta \phi_{12}\right|_{\operatorname{moxt} \text { tip }}=\left(l_{1}-l_{2}\right) \psi_{\mathrm{tm}}+\left(m_{1}-m_{2}\right) \psi_{\mathrm{ss}}$, with the Gouy phase shifts $\psi_{\operatorname{man}}$ and $\psi_{\mathrm{sm}}$ solely determined by the geometry of the cavity. Since the Gouy phase shift depends only on the cavity geometry and is independent of wavelength, it corresponds to a pure carrier-envelope offset frequency shift, viz.

$$
f_{C E O, 1}-f_{C E O, 2}=\left.\frac{f_{\text {rep }}}{2 \pi} \Delta \phi_{12}\right|_{\text {round trip }}
$$

Thus, by coupling combs to the higher-order modes of an optical cavity they naturally phase cycle, generating new combs which can also be made resonant. As we discuss in the next section, mode-matching also provides spatial isolation of the signal analogous to noncollinear phase matching in conventional 2D spectrometers.

\section{One Cavity Implementation}

The spatial-mode selectivity of the cavity is analogous to phase matching in conventional 2D spectroscopy setups. Just as one isolates a desired signal in a boxcar geometry by detecting in a certain direction, in CE-2D spectroscopy using higher-order modes one can isolate a desired signal by detecting in a certain spatial mode. The generation and resonant enhancement of CE-2D signals using higher order cavity modes can thus be viewed as selecting a desired third-order response signal through a combination of both phase cycling and spatial discrimination/phase matching. This combination can make CE-2D spectroscopy highly selective, even in a completely collinear geometry, since both the cavity and the detection methods can discriminate against undesired signals. In figure 1, we show a simple signal isolation scheme based on a combination of spatial-mode discrimination and frequency discrimination. The input fields $E_{1}, E_{2}$, and $E_{3}$ are coupled to the $\mathrm{TEM}_{11}, \mathrm{TEM}_{10}$, and the $\mathrm{TEM}_{01}$ modes, respectively, generating a rephasing signal in the lowest-order $\mathrm{TEM}_{00}$. Light from the cavity is then launched in a single-mode fiber to remove the $E_{1}, E_{2}$, and $E_{3}$ fields from the beam. Also coupled to the fiber is an intense local oscillator frequency comb with $f_{\mathrm{CEO}, L O}$ near $f_{\mathrm{CEO}}{ }^{\left.{ }^{3}\right)}$. After exiting the fiber, the modulation on the light at frequency $f_{\mathrm{CEO}, L O}-f^{(3)}$ is detected using a lock-in spectrometer, providing discrimination in the frequency domain. For waiting times $T>0$, this scheme additionally discriminates in the time domain due to the fact that the local-oscillator does not overlap temporally with $E_{1}$ and $E_{2}$. 
a)

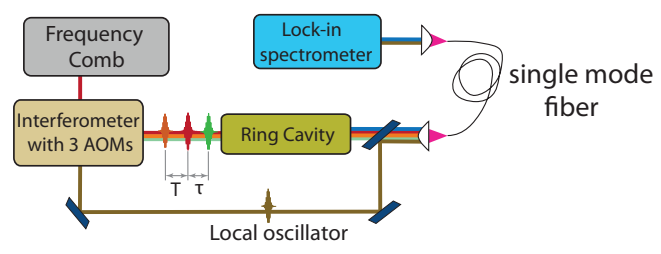

b) $\mathrm{TEM}_{11}^{10} \downarrow 01 \quad 00$

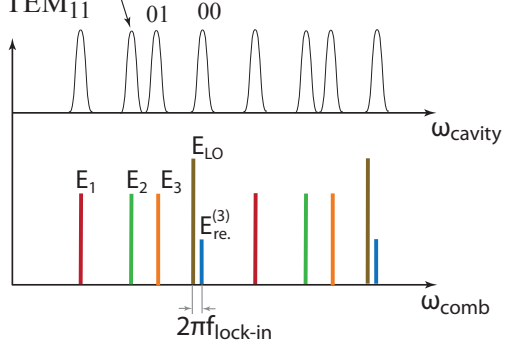

Fig. 1 The generated frequency comb $E^{(3)}$ is different from the three driving fields in time, spatial mode, and frequency. The proposed scheme shown here uses all three to isolate the 2D signal from background. First, the TEM 00 beam from the cavity is selected by coupling the cavity output to a single mode fiber. Second, heterodyne detecting the signal with a local oscillator (LO) frequency comb and a lock-in spectrometer discriminates against the collinear combs $E_{1}, E_{2}$, and $E_{3}$ in the frequency domain, since their heterodyne signals with the LO comb appear at the wrong frequencies. Third, background signals due to $E_{1}$ and $E_{2}$ are further suppressed by adjusting the delay of the LO pulses such that they coincide temporally with only the $E^{(3)}$ and $E_{3}$ combs.

\section{References}

1. S. Y. T. van de Meerakker, H. L. Bethlem, and G. Meijer, Nature Physics 4, 595-602 (2008).

2. L. J. G. W. vanWilderen and J. Bredenbeck, Angewandte Chem. Int. Ed. 54, 11,62411,640 (2015). 3. C. M. Lovejoy and D. J. Nesbitt, J. Chem. Phys. 86, 3151-3165 (1987).

4. G. Gagliardi and H.-P. Loock, eds., Cavity Enhanced Spectroscopy and Sensing (Springer, 2013). 5. M. A. R. Reber, Y. Chen, and T. K. Allison, Optica 3, 311-317 (2016).

6. B. Lomsadze and S. T. Cundiff, Science 357, 1389-1391 (2017).

7. T. K. Allison, J. Phys. B 50, 044,004 (2017). 